\title{
Dense arrays of micro-needles for recording and electrical stimulation of neural activity in acute brain slices
}

\author{
D E Gunning ${ }^{1,7}$, J M Beggs ${ }^{2}$, W Dabrowski ${ }^{3}$, P Hottowy ${ }^{3,4}$, C J Kenney ${ }^{5}$, \\ A Sher ${ }^{4}$, A M Litke ${ }^{4,6}$ and K Mathieson ${ }^{1,6}$ \\ ${ }^{1}$ IOP, University of Strathclyde, Glasgow G4 0NW, UK \\ ${ }^{2}$ Biocomplexity Institute, University of Indiana, Bloomington, IN 47405, USA \\ ${ }^{3}$ Faculty of Physics and Applied Computer Science, AGH University of Science and Technology, \\ Krakow, 30-059, Poland \\ ${ }^{4}$ SCIPP, University of California Santa Cruz, Santa Cruz, CA 95064, USA \\ ${ }^{5}$ SLAC National Accelerator Laboratory, Menlo Park, CA 94025, USA \\ E-mail: deborah.gunning@strath.ac.uk
}

Received 22 August 2012

Accepted for publication 13 November 2012

Published 12 December 2012

Online at stacks.iop.org/JNE/10/016007

\begin{abstract}
Objective. This paper describes the design, microfabrication, electrical characterization and biological evaluation of a high-density micro-needle array. The array records from and electrically stimulates individual neurons simultaneously in acute slices of brain tissue. Approach. Acute slices, arguably the closest in-vitro model of the brain, have a damaged surface layer. Since electrophysiological recording methods rely heavily on electrode-cell proximity, this layer significantly attenuates the signal amplitude making the use of traditional planar electrodes unsuitable. To penetrate into the tissue, bypassing the tissue surface, and to record and stimulate neural activity in the healthy interior volume of the slice, an array of 61 micro-needles was fabricated. Main results. This device is shown to record extracellular action potentials from individual neurons in acute cortical slices with a signal to noise ratio of up to $\sim 15: 1$. Electrical stimulation of individual neurons is achieved with stimulation thresholds of 1.1-2.9 $\mu$ A. Significance. The novelty of this system is the combination of close needle spacing $(60 \mu \mathrm{m})$, needle heights of up to $250 \mu \mathrm{m}$ and small $(5-10 \mu \mathrm{m}$ diameter) electrodes allowing the recording of single unit activity. The array is coupled to a custom-designed readout system forming a powerful electrophysiological tool that permits two-way electrode-cell communication with populations of neurons in acute brain slices.
\end{abstract}

(Some figures may appear in colour only in the online journal)

\section{Introduction}

The study of interactions between neurons in the brain is of scientific importance and has many applications including advancing the understanding of retinal processing [1], network development [2] and neurodegeneration [3, 4].

\footnotetext{
${ }^{6}$ Equal contribution.

${ }^{7}$ Author to whom any correspondence should be addressed.
}

Current veins of research revolve around trying to record and stimulate with optimal spatial and temporal resolution, activity in networks of neurons throughout a useful volume of tissue. Many approaches have been adopted including optical and electrical techniques, in vitro and in vivo. Optical methods include imaging neurons' activity using voltage sensitive [5] or calcium indicator dyes [6] coupled with optical recording techniques. At best, these methods offer individual spike resolution, limiting the efficacy of neuron detection. 
In this paper, an electrophysiological method of recording and stimulating neurons in acute brain slice preparations is described. Electrophysiological methods have been employed very successfully for many years. A general disadvantage is that the necessary placement of electrodes in close proximity to cells makes it a relatively invasive method. This is outweighed, however, by the critical combination of high spatial and temporal resolution that recordings from planar microelectrode array technologies afford and that are essential for reliable and high efficiency detection of individual neurons and action potentials. Planar arrays of microelectrodes can successfully record neuronal action potentials in vitro from retina $[1,7]$ and cultures (dissociated and organotypic) [8-11] and in acute brain slice preparations [12] although local field potentials are far more commonly recorded $[13,14]$. The planar nature of these types of arrays limits their usefulness with acute in vitro brain preparations since the surface layer of the tissue is damaged during the slice preparation. For studying acute slices, electrophysiologists have used, for many decades, a patch-clamp $[15,16]$ to attach to, intracellularly record from and stimulate a single neuron. The very nature of the patchclamp technique restricts its use to studying the behaviour and connectivity of only a limited number of cells. To study the behaviour of many (tens or hundreds of) neurons in acute local networks, arrays of micro-needles have been developed to penetrate past the damaged surface layer. Processes have been devised by several groups for the fabrication of needle arrays for both in vivo and in vitro preparations and for recording single and multi-cell activity and local field potentials [17-21]. The current state-of-the-art in devices of this nature, whilst working very well to record and stimulate neurons in acute (and in vivo) neural preparations, have widely spaced needles and as a result, limited spatial resolution or they have limited needle lengths with little flexibility in the fabrication process for multiple lengths. Current devices include 3D micromolded microelectrode arrays (700 $\mu \mathrm{m}$ spacing [17]), a high-aspect ratio micro-needle array $(300 \mu \mathrm{m} \times 400 \mu \mathrm{m}$ spacing [18]) and commercially available technologies such as the Utah array (400 $\mu \mathrm{m}$ spacing [19]), the bioMEA ${ }^{\mathrm{TM}}$ system (250 $\mu \mathrm{m} \times 450 \mu \mathrm{m}$ spacing [20]) and the Qwane Biosciences MEA biochip ( $60 \mu \mathrm{m}$ high needles with $100 \mu \mathrm{m}$ spacing [21]).

The focus of the work presented in this paper has been the development and characterization of an array of micro-needles for in vitro acute slice work, which aims to address the aforementioned limitations of other currently available technologies by providing an array of closely spaced needles of substantial length. The first generation high-density array of micro-needles enables the combination of unprecedented spatial and temporal resolution and the flexibility to incorporate multiple length needles. The device is designed to be compatible with existing low noise preamplification and stimulation circuitry $[22,23]$. The array itself is non-transparent and resembles a microscopic bed-of-nails. It is comprised of 61 hexagonally close-packed needles, with $60 \mu \mathrm{m}$ inter-needle spacing. Each needle can be engineered to measure up to $250 \mu \mathrm{m}$ in length with the possibility of multiple needle lengths on any given array, and has a small (5-10 $\mu \mathrm{m}$ diameter) conducting tip. This results in an array of closely spaced, low impedance, electrodes capable of penetrating into neural tissue. Localized recordings of individual neuronal behaviour were obtained with a good signal to noise ratio and high spatial resolution. To quantify this statement: a typical electrode has an impedance of $300 \mathrm{k} \Omega$ at $1 \mathrm{kHz}$ and an average RMS noise of $\sim 5 \mu \mathrm{V}$ in the frequency bandpass range of 43-2000 Hz. Action potentials were recorded (with amplitudes up to $110 \mu \mathrm{V}$ ) from acute rat cortical slices using an array with $100 \mu \mathrm{m}$ length needles. Accounting for both the electronic noise of the system $(\sim 5 \mu \mathrm{V}$ RMS $)$ and the biological background noise which varies depending on the nature of the surrounding neural activity (i.e. it is higher when many cells have bursting activity simultaneously), the system has $\sim 7 \mu \mathrm{V}$ RMS noise and typically operated with a signal to noise ratio of up to $\sim 15: 1$. The electrodes were also shown, as proof of principle, to be capable of eliciting action potentials with individual neuron precision via electrical stimulation using three-phase charge-balanced current pulses with $50 \mu$ s/phase and current amplitudes as low as $\sim 1.1 \mu \mathrm{A}$.

\section{Materials and methods}

\subsection{Electrode array fabrication}

The bed-of-nails is made using a novel combination of semiconductor fabrication techniques. It is a double-sided process which relies on the deep reactive ion etching (DRIE) of silicon, low-pressure chemical vapour deposition of tungsten (for conformal coating of high aspect ratio structures), selective chemical wet etching for wafer thinning and photolithographic techniques to pattern positive tone photoresists. The process is outlined in steps (i) to (viii) and these correspond to the schematics in figure 1.

(i) DRIE etch silicon. The first step in the fabrication process is to etch arrays of 61 hexagonally close packed, high aspect ratio (11:1), tapered holes to a depth of $\sim 300 \mu \mathrm{m}$ in to a 4-inch diameter, $350 \mu \mathrm{m}$ thick silicon wafer. On to this wafer, ten devices are fitted. Following a combined pre-bake at $150{ }^{\circ} \mathrm{C}$ (to remove moisture) and an HMDS (hexamethyldisilazane) prime (to improve adhesion of photoresist to the wafer), SPR220-7 photoresist is spun to a thickness of $7 \mu \mathrm{m}$, and baked on a hotplate at $90{ }^{\circ} \mathrm{C}$ for $200 \mathrm{~s}$. Standard UV contact photolithography (using developer MF-26A) is used to pattern the photoresist in to arrays of holes, each having a $25 \mu \mathrm{m}$ diameter. This patterned mask is hardened by oven baking for $1 \mathrm{~h}$ at $120^{\circ} \mathrm{C}$. The holes are etched using a STS ICP (inductively coupled plasma) deep reactive ion etcher, which switches between $\mathrm{CH}_{4}$ passivation and $\mathrm{SF}_{6}$ etch steps to achieve the required etch profile (Bosch process). This profile defines the shape of the needle.

(ii) Thermal oxidation and metallization. A $2 \mu \mathrm{m}$ thick silicon dioxide layer is thermally grown at $1050{ }^{\circ} \mathrm{C}$ to form a high-quality, uniform sidewall insulation. The tapered point at the base of the etched hole will eventually be the conducting tip of the needle making it important to have a conformal metal coating. To this end, $400 \mathrm{~nm}$ of LPCVD 


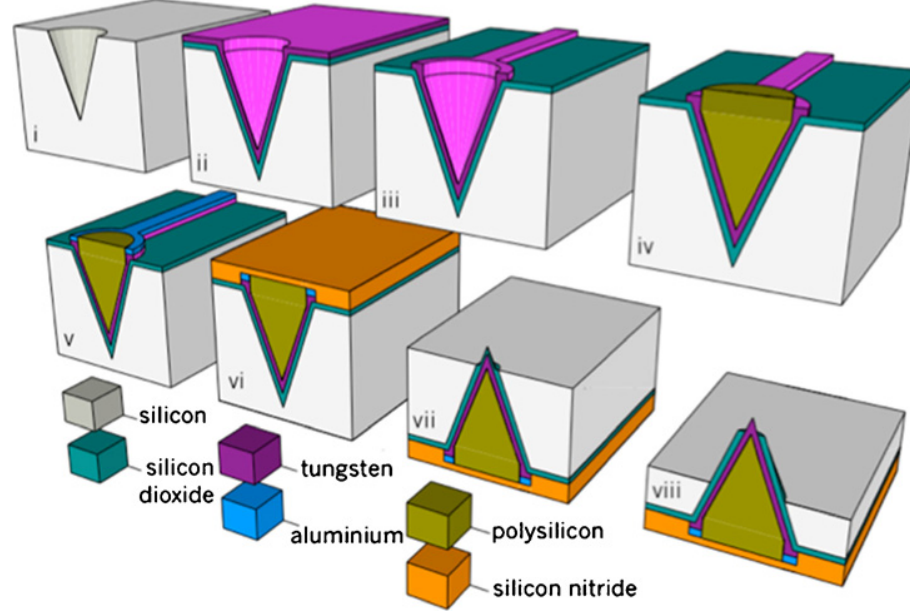

(i) Silicon etch (DRIE)

(ii) Thermal oxidation and metallization

(iii) Readout lithography

(iv) Polysilicon deposition

(v) Aluminium deposition and patterning

(vi) Silicon nitride deposition and patterning

(vii) Silicon and tip oxide etch

(viii) Final silicon and tip oxide etches

Figure 1. A schematic of process steps involved in the fabrication of micro-needle arrays. The process is illustrated for the cross section of a single needle. A detailed explanation for each step is given in section 2.1.

(low pressure chemical vapour deposition) tungsten (W) is deposited. Prior to the tungsten deposition, a titanium layer $(50 \mathrm{~nm})$ was sputtered to promote tungsten adhesion to the thermal silicon dioxide (not shown in figure 1).

(iii) Readout lithography. The tungsten layer is patterned using a pre-bake and HMDS prime followed by spinning Shipley 3612 photoresist to a thickness of $1.6 \mu \mathrm{m}$. The photoresist is oven baked at $90{ }^{\circ} \mathrm{C}$ for $30 \mathrm{~min}$ before UV exposure and oven baked at $120{ }^{\circ} \mathrm{C}$ for $60 \mathrm{~min}$ after developing. Photoresist spun over the perforated surface of the silicon wafer is always spun relatively thin. It is baked in an oven rather than a hotplate since lower gradient temperature ramping avoids resist bubbling over the etched holes. This resist layer is used as standard for all of the remaining photolithography steps in this process. An $\mathrm{SF}_{6}$ reactive-ion etch removes the exposed tungsten, electrically isolating each electrode.

(iv) Polysilicon deposition. For mechanical strength, $4 \mu \mathrm{m}$ of LPCVD polysilicon is deposited and patterned to fill, or at least partially fill, the holes. A $1.6 \mu$ m photoresist mask and an $\mathrm{SF}_{6}$ etch with $10 \%$ oxygen are used respectively to mask and etch the polysilicon. This exposes the tungsten readout for electrical contact in the next step. These first steps have essentially embedded arrays of conducting needles in to the wafer.

(v) Aluminium (Al) deposition and patterning. A short argon etch removes a few nanometres of surface material preparing the wafer surface for a $1 \mu \mathrm{m}$ layer of sputter deposited aluminium, which makes electrical contact with the exposed tungsten. Sputtered aluminium is a wellestablished, reliable contact for wire bonding (which is used to connect the array to the readout electronics at a later stage). The aluminium is patterned using a $1.6 \mu \mathrm{m}$ photoresist mask and a Al-11 chemical wet etch (premixed phosphoric, acetic and nitric acid in water) heated to $40{ }^{\circ} \mathrm{C}$. The combination of tungsten and aluminium form an electrical connection between the tungsten tip of each needle (the electrode) and an aluminium bond pad. See the right hand image of figure 2 (b) for further reference.
The backside of an array is photographed showing the readout from 61 electrodes.

(vi) Silicon nitride deposition and patterning. The aluminium/ tungsten tracks are protected with a $500 \mathrm{~nm}$ layer of low temperature $\left(350^{\circ} \mathrm{C}\right) \mathrm{PECVD}$ (plasma enhanced chemical vapour deposition) silicon nitride. The following steps are not shown in figure 1 . To expose the aluminium bond pads, the silicon nitride is selectively etched (RIE) using $\mathrm{SF}_{6}$ and $10 \%$ oxygen. The wafer is then diced to release individual devices.

(vii) Silicon and tip oxide etch. Two etch masks, one on each side of the device, are required before etching hundreds of microns of bulk silicon to expose the array of needles. In this process, the bulk silicon will ultimately be etched to leave $\sim 100 \mu \mathrm{m}$ thickness (this provides mechanical strength around the needles). To retain the mechanical strength of the device, a frame around the edge of the device is protected with a mask of silicon dioxide. On the backside (with holes and readout) of each device, Protek $\mathrm{B} 3$, an etch mask resistive to wet chemical base etches is spin-coated.

A $25 \%$ concentration TMAH (tetra methyl ammonium hydroxide in water) solution, heated to $95{ }^{\circ} \mathrm{C}$, is used to remove enough of the bulk silicon to expose the oxide-coated needles. The silicon etch rate is $\sim 0.9 \mu \mathrm{m} \mathrm{min}^{-1}$ and so thin $(325-350 \mu \mathrm{m})$ silicon wafers are used to avoid long etch times. The slow etch rate is beneficial for accurately defining the length of the needle and, in particular, the conductive tip. At some point during the silicon etching, the arrays of oxidecoated needles will begin to appear. When the desired length of tip (length of needle needed to be conducting) is exposed from the silicon, the device is removed from the TMAH and rinsed thoroughly in water. Using dilute (5\%) buffered hydrofluoric acid, as much as $75 \%$ of the tip oxide thickness is etched. It is critical that a thin layer of oxide remains at the tip and no tungsten is exposed.

(viii) Final silicon and tip oxide etch. The silicon etch is continued to expose the required length of the needle 
(a)

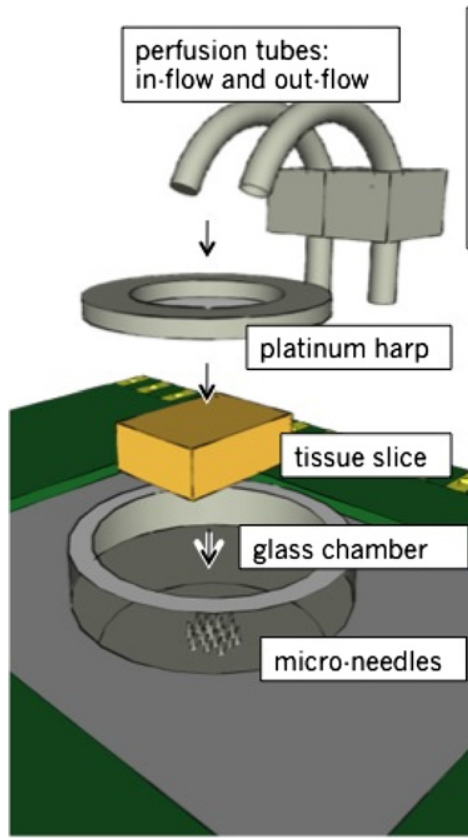

(b)

front-side:

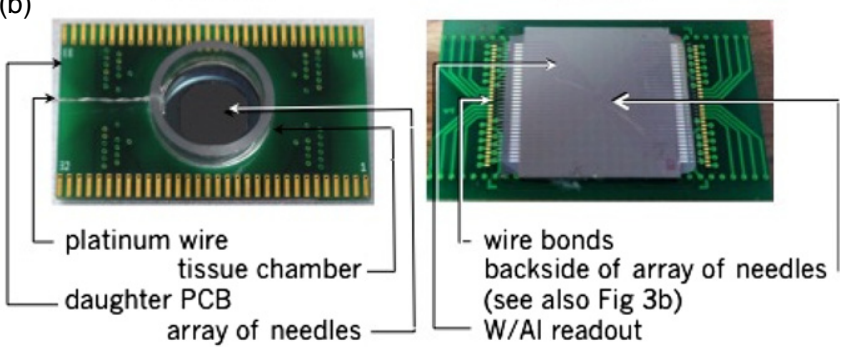

(c)

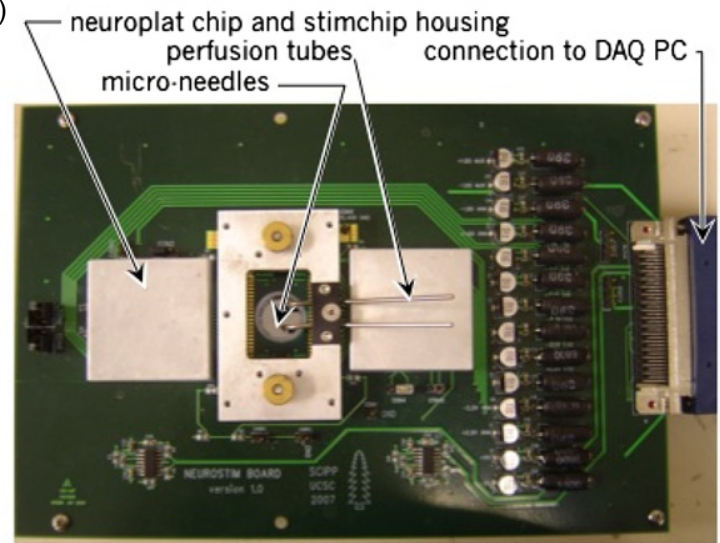

Figure 2. Schematic (a) of the array, tissue, harp and perfusion interface (not to scale). (b) Photo of the front- (left) and back- (right) side of the micro-needle array wire-bonded into a daughter PCB compatible with existing readout and stimulation electronics. As shown in the left-hand image, on the surface of the silicon substrate, surrounding the array of needles, a $10 \mathrm{~mm}$ diameter glass chamber has been glued with a grounding platinum wire attached around its inside circumference. The right-hand image shows the electrical (tungsten/ aluminium) readout from each electrode to each bond pad and the wire-bonds connecting the array to the daughter PCB. (c) Photo of the readout board with the micro-needle array connected to the readout and stimulation electronics through a $z$-axis connector. Perfusion tubes allow the flow of physiological solution through the tissue chamber.

electrode, leaving a few tens of microns of silicon as the support substrate. A $2 \%$ concentration of hydrofluoric acid (which is selective to tungsten) is used to remove the remaining oxide from the tip, exposing a small tungsten electrode. The protective Protek is removed in acetone followed by a low-power oxygen plasma clean. The silicon chip is glued to a support piece of silicon or glass (for strength) and is wire-bonded to a PCB making it compatible with the existing readout system (see figure 2 ).

The final step of fabrication, not shown in figure 1, is to lower the impedance of the electrode. This is done by electroplating the tungsten needle tips in chloroplatinic acid at a current density of $4 \mathrm{nA} \mu \mathrm{m}^{-2}$ causing the formation of a granular platinum structure (platinum black). The electrode surface area is significantly increased but with negligible increase to the electrode diameter.

\subsection{Readout and stimulation electronics}

The array is wire bonded to a daughter PCB (see figure 2(b)) enabling it to interface with an existing circuit board (see figure 2(c)) housing low-noise readout and low-artefact electrical stimulation electronics [22, 23]. The bed-of-nails device connects to the electronics through $z$-axis interconnects. Two 64-channel readout ASICs (Neuroplat-chip [24]) accouple, amplify, bandpass-filter and multiplex recorded signals from the array at $20 \mathrm{kHz}$ per channel. Arbitrary patterned electrical stimulation is possible through two computercontrolled ASICs (Stim-chip [23, 25]). Only 32 channels of each chip are used in this application. Whilst the needle separation defines the spatial resolution of the system $(60 \mu \mathrm{m})$, the electronics define the temporal resolution $(50 \mu \mathrm{s})$ completing this 3-d neural recording and stimulation system for acute slices.

\subsection{Experimental setup}

This section describes the procedure and experimental setup for the preparation of acute slices and the running of experiments. A schematic of the experimental set-up is shown in figure 2. A clamp incorporating flow tubes and an in-line heater is used for the perfusion of artificial cerebrospinal fluid (ACSF) which is made up of $124 \mathrm{mM} \mathrm{NaCl}, 3 \mathrm{mM}$ $\mathrm{KCl}, 2 \mathrm{mM} \mathrm{MgSO}_{4}, 1.25 \mathrm{mM} \mathrm{NaH} \mathrm{PO}_{4}, 26 \mathrm{mM} \mathrm{NaHCO}$, $10 \mathrm{mM}$ D-glucose and $2 \mathrm{mM} \mathrm{CaCl}_{2}$. ACSF closely matches the electrolyte concentrations of cerebrospinal fluid (CSF) and is commonly used in in vitro neural studies to imitate the natural environment of neurons [26, 27]. A reference platinum wire encircles the chamber and is the differential input to the amplifier channels. The needles are highlighted in the centre of the chamber.

Animals were maintained in the animal facility at the University of California Santa Cruz (UCSC) and used in accordance with protocols approved by the UCSC Institutional Animal Care and Use Committee. Sprague-Dawley rats 
(a)

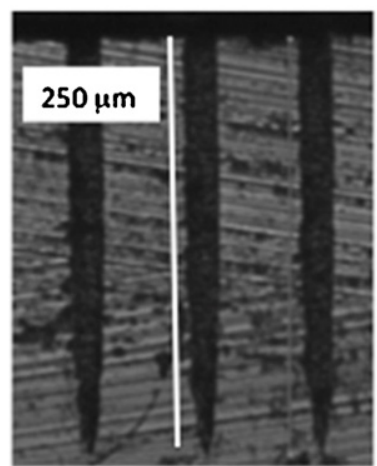

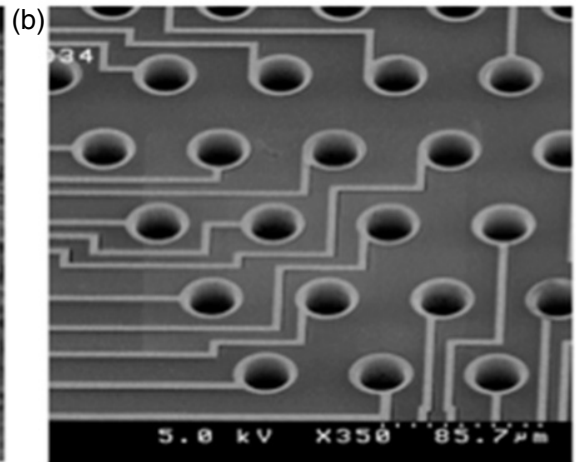

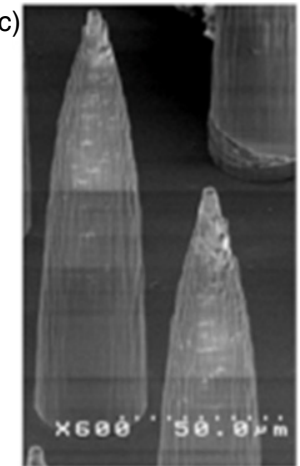

(d)
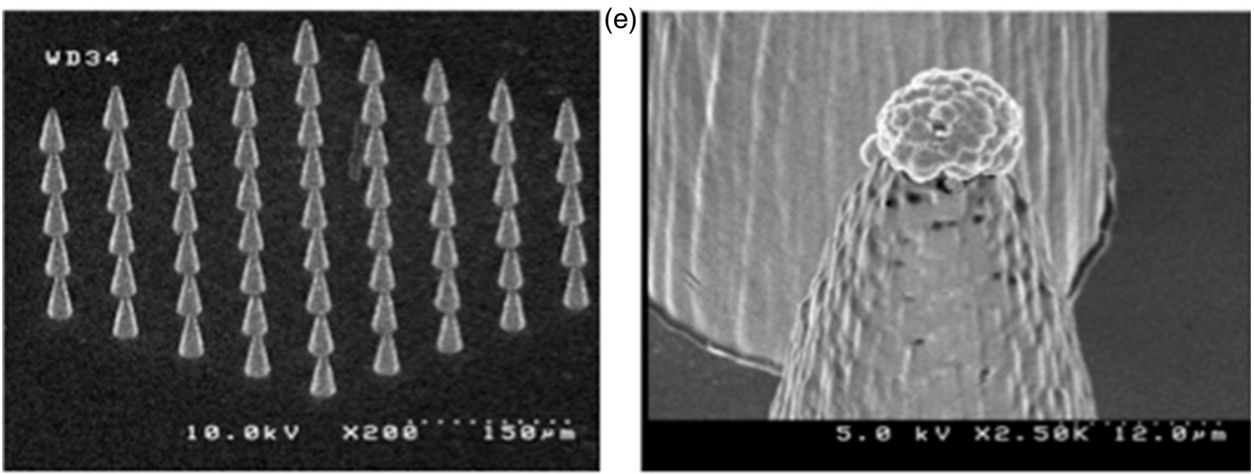

Figure 3. A series of images from various stages in the fabrication of a micro-needle array. (a, b) Cross section and surface image, respectively, of etched holes complete with conformal thermal oxide and patterned LPCVD tungsten. (c) Mechanical sample of needles which are $\sim 150 \mu \mathrm{m}$ in height. The base diameter is $30 \mu \mathrm{m}$ and the tip, which in this case is still insulated with silicon dioxide, is a few microns in diameter. It illustrates the tapered profile created by the DRIE silicon etching. (d) A complete, working array of 61 hexagonally close-packed needles with an inter-needle spacing of $60 \mu \mathrm{m}$ and $~ 50 \mu \mathrm{m}$ needle length. (e) A single needle tip on which the tungsten has been exposed and subsequently electroplated with platinum black.

(purchased from Charles River Laboratory) aged between p14 and p21 were used. The intact brain is removed and $300 \mu \mathrm{m}$ thick slices were prepared (using a microtome) in a sucrose solution (206 mM sucrose, $2 \mathrm{mM} \mathrm{KCl,} 1 \mathrm{mM} \mathrm{MgCl} 2,2 \mathrm{mM}$ $\mathrm{MgSO}_{4}, 1.25 \mathrm{mM} \mathrm{NaH} \mathrm{PO}_{4}, 26 \mathrm{mM} \mathrm{NaHCO}, 10 \mathrm{mM}$ Dglucose, $1 \mathrm{mM} \mathrm{CaCl}$ ) chilled to $4{ }^{\circ} \mathrm{C}$. The slices were cut using the method described in [28]. A slice is incubated in ACSF for approximately $1 \mathrm{~h}$ at room temperature and is then transferred on to the array and positioned over the needles. The data presented here is taken from 7 slices harvested from 6 brains.

A platinum wire, framing a fine mesh membrane, weights the tissue to the array. Excited ACSF (eACSF) solution is perfused through the chamber to maintain tissue health. The solution closely resembles the ACSF solution described above but has the magnesium element $\left(\mathrm{MgSO}_{4}\right)$ removed and potassium levels elevated to $5 \mathrm{mM}$ to encourage cell activity. It is perfused at a rate of $3 \mathrm{ml} \mathrm{min}^{-1}$ at a temperature of $37^{\circ} \mathrm{C}$ and is bubbled throughout experiments with a $95 \% \mathrm{O}_{2}: 5 \%$ $\mathrm{CO}_{2}$ gas mix.

\section{Results}

\subsection{Device fabrication}

A process was successfully developed for the fabrication of the high-density micro-needle arrays. Figure 3 shows a variety of microscope and SEM images of the micro-needle array at various stages of the fabrication process.

\subsection{Electrical characterization}

In order to record action potentials from individual neurons, a microelectrode must have low impedance whilst maintaining a small diameter. The electrode impedance is measured by applying a sinusoidal voltage stimulus $(0.1 \mathrm{~V}$ peak-to-peak $)$ through a physiological saline solution. The measurement of interest is at $1 \mathrm{kHz}$ since typical extracellular action potentials have $\sim 1$ ms duration. The impedance of the electrodes was measured before tungsten exposure, before platinizing and after platinizing and was found to lower, from $\sim 1 \mathrm{M} \Omega$ after oxide removal, to $\sim 300 \mathrm{k} \Omega$ after platinizing. For stimulating electrodes it is also important to quantify the charge capable of being delivered by each electrode and thereby its suitability for cell stimulation. Using cyclic voltammetry, the charge capacity of a platinum black electrode is found to measure in the region of $0.1 \mathrm{mC} \mathrm{cm}^{-2}$ consistent with values from the literature [29].

\subsection{Electrophysiological recordings}

Experiments were performed with rats aged between postnatal day 14 and 21. For the data shown in this section, the slice was cut from a p19 Sprague-Dawley rat. Action potentials were recorded from cortex within the first $10 \mathrm{~min}$ of placing the slice on the array and starting the perfusion of oxygenated 


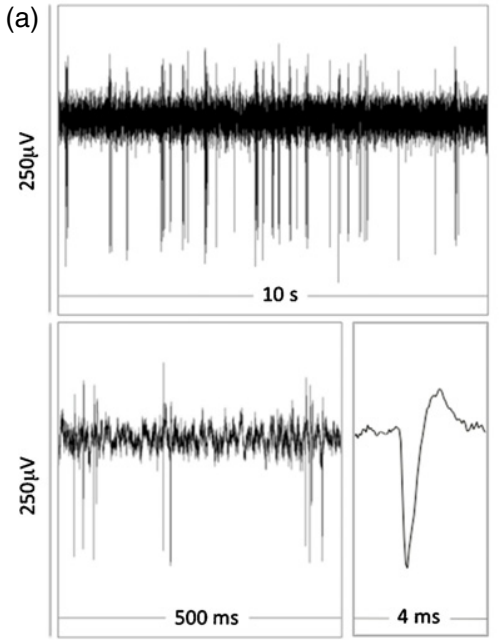

(b)

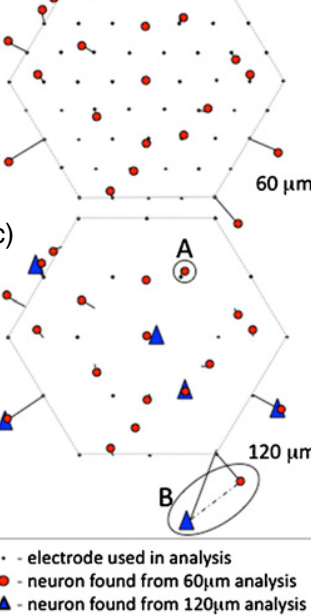

Figure 4. Examples of action potentials recorded on a single electrode from neurons in an acute slice of rat cortex are shown in (a) over a $10 \mathrm{~s}, 500 \mathrm{~ms}$ and $4 \mathrm{~ms}$ time period. In (b), the $60 \mu \mathrm{m}$ spaced electrode map is plotted (electrodes are black dots) with the positions of 19 identified neurons indicated with a red circle. In (c), the data is sub-sampled to indicate the identified neurons from an array with $120 \mu \mathrm{m}$ electrode spacing. The six neurons identified are indicated by blue triangles. The neurons found for the $60 \mu \mathrm{m}$ spacing analysis are overlaid on the $120 \mu \mathrm{m}$ spacing plot. Solid lines connect neurons to the electrode on which they were identified. In cases where a neuron in common with both analyses is found in two different locations, a dashed line connects the two neurons. A and $\mathrm{B}$ are referred to in section 4 .

(95\% oxygen: 5\% carbon dioxide) eACSF. Typical spike amplitudes (extracellular) were between 30 and $110 \mu \mathrm{V}$. Data were recorded for $30 \mathrm{~min}$ and spike sorted using the principal component analysis/clustering techniques described in [7] to identify the number, position and activity of the neurons.

Examples of extracellular action potentials recorded from a 61-needle array with a $60 \mu \mathrm{m}$ inter-electrode spacing and $100 \mu \mathrm{m}$ needle height are shown in plot (a) of figure 4. A 30 min recording of spontaneous activity was analysed and identified 19 individual neurons. Although neural activity is recorded across all electrodes and from more neurons, a strict spike-sorting regime [7] reduces the data to 19 individual (and very cleanly identified) single units as shown in figure 4(b). The position of each neuron is defined as the centre point of the two-dimensional Gaussian fit to the electrophysiological image (EI) where an EI is the image of each identified neuron in terms of the averaged voltage waveform generated on each electrode as the neuron spikes. The averaging (which is over 1000 spikes in this analysis) eliminates uncorrelated noise and reveals the somatic signals as well as the smaller dendritic or axonal signals (see [7]). The method of positioning the neuron, by fitting to the EI, can position a neuron outside of the bounds of the array but the accuracy is reduced since less data exists for these neurons. A line attaches each neuron to the seed electrode where the seed electrode is defined as the electrode that records the signal of largest amplitude, usually the electrode closest to the cell body. To highlight the advantage of high-density electrodes, the data were re-analysed with $75 \%$ of the electrodes removed to have,
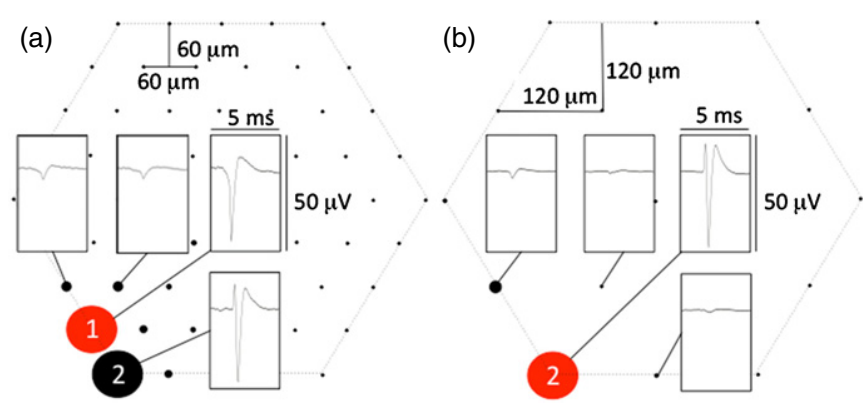

Figure 5. The EIs from a neuron detected in both (a) the $60 \mu \mathrm{m}$ and (b) the sub-sampled $120 \mu \mathrm{m}$ analyses (but on different electrodes) are shown.

effectively, an array with $120 \mu \mathrm{m}$ inter-electrode spacing and a hexagonally close-packed geometry. The data were analysed using every permutation of the $120 \mu \mathrm{m}$ spaced array (there are 4) and the mapping with the most competitive result was used as a comparison to the $60 \mu \mathrm{m}$ spaced array. In figure 4(c), the positions of neurons found from both analyses (60 and $120 \mu \mathrm{m})$ are plotted. Sub-sampling the data in this manner reduces the number of neurons by a factor of 3.2 (from 19 to 6), while the number of neurons per electrode remains the same, from 0.31 to 0.32 . A further six unique datasets (from different tissue preparations) were also analysed this way. It was found that the number of neurons, at best, was halved when the electrode spacing was increased by the factor of two. At worst, the number of neurons was reduced to less than $10 \%$.

In figure 5, a further example of the benefit of higher spatial resolution is illustrated. The EI generated for a neuron identified in both analyses $(60 \mu \mathrm{m}$ and $120 \mu \mathrm{m})$ but on different electrodes is shown. From the triphasic signal recorded on electrode 2 it is clear that the same neuron has been identified. However, it is also clear that information has been lost e.g. the somatic (biphasic) signal on electrode 1 is not observed in the subsampled analysis.

Figure 6 displays each of the 61 hexagonally closepacked electrodes as a circle with a diameter representative of the maximum (negative) signal amplitude (from a particular neuron) recorded on each electrode-the EI of the neuron. The diameter of the circle is not exactly proportional to the amplitude since it saturates at the pitch of the electrodes to avoid overlapping circles in the plot. In this figure, the EIs of four neurons are shown. The seed electrode, coloured red, is represented by the circle of largest diameter for each of the four neurons. The average waveform recorded by the seed electrode on all four neurons is shown. For neurons A and C, waveforms from neighbouring electrodes are shown also. On all 4 neurons, a classic extracellular somatic waveform can be seen on the seed electrode. On neighbouring electrodes, much smaller triphasic signals are observed which may be associated with dendritic or axonal signals. A total of 19 neurons were found over an electrode coverage area of $0.13 \mathrm{~mm}^{2}$.

\subsection{Electrical stimulation of neurons}

Electrical stimulation is a critical component of network studies. Electrodes, whether single wire or arrayed 


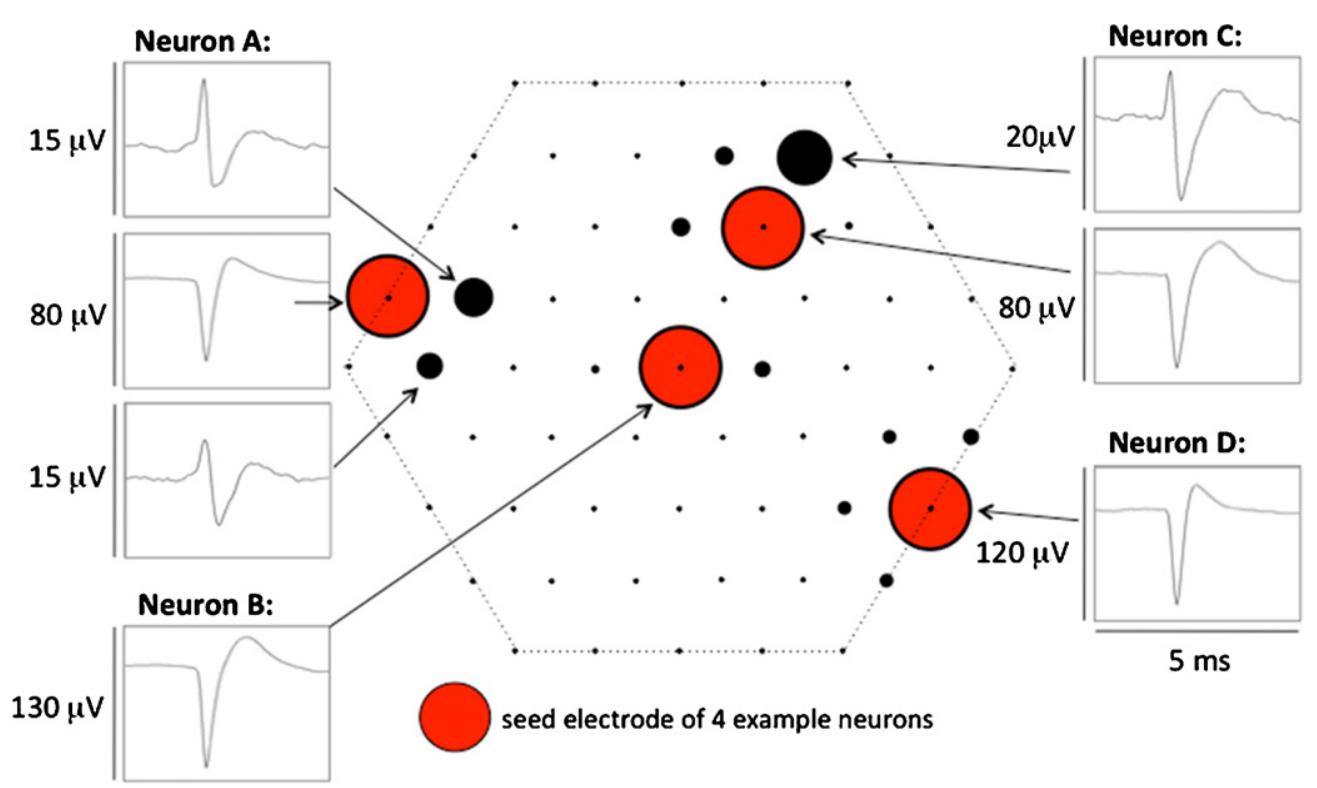

Figure 6. The electrophysiological images of four individual neurons (A-D) identified from their spontaneous activity as recorded on a 61-electrode micro-needle array.

\section{Neuron 1:}
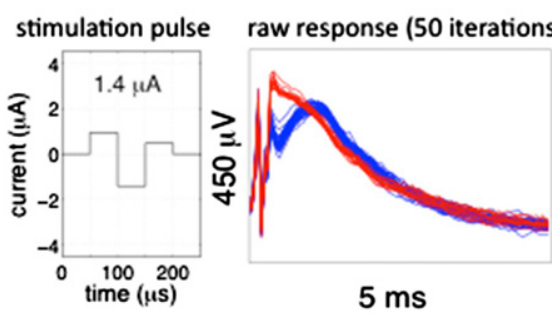

Neuron 2:
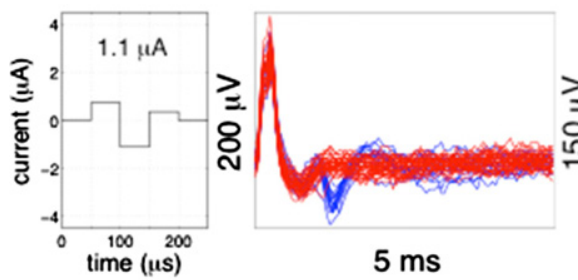
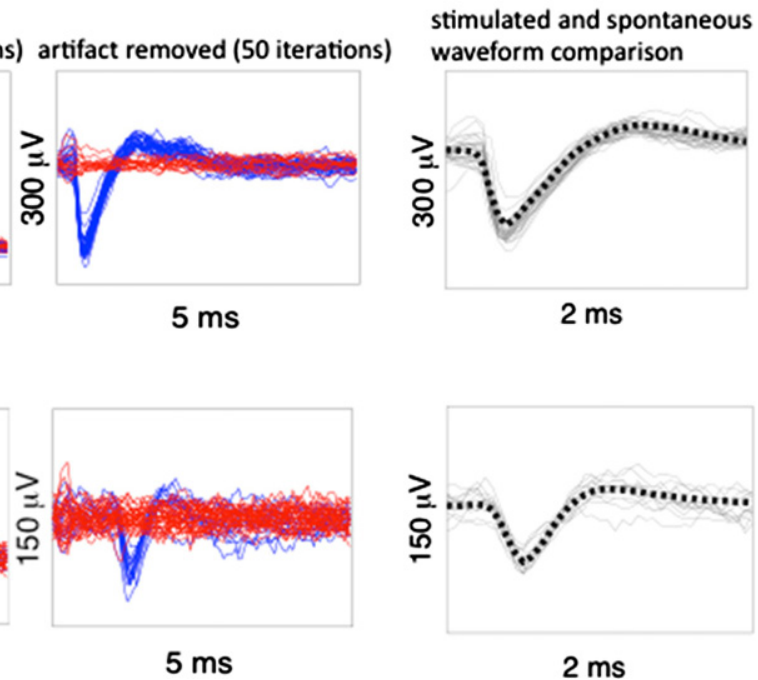

$5 \mathrm{~ms}$
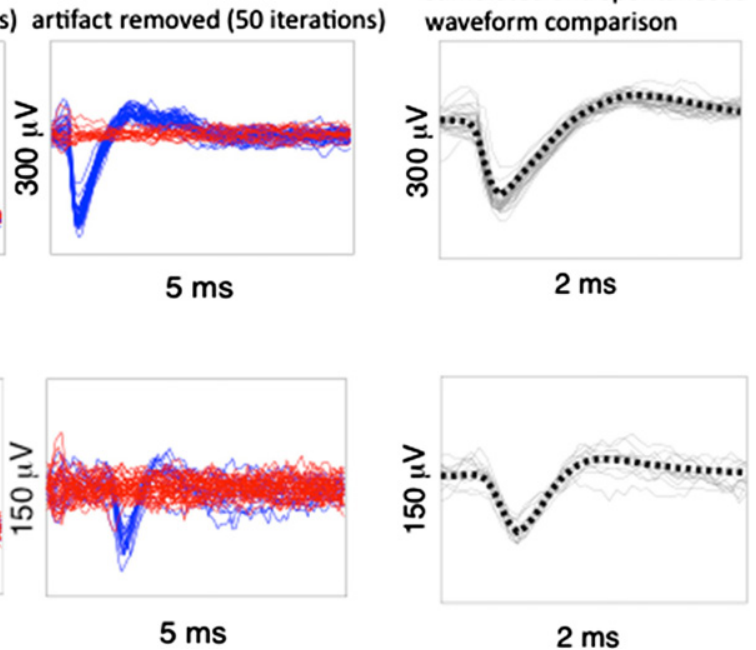

Figure 7. For two neurons, responses to electrical stimulation are shown. In the first column the charge-balanced, triphasic stimulation pulse is shown. $50 \mu \mathrm{s}$ after this pulse is applied, a $5 \mathrm{~ms}$ recording is shown for each neuron (second column). The iterations where a response is recorded are highlighted in blue. Trials with no response are highlighted red. In the third column, the stimulation artefact has been removed from the raw data by identifying the artefact waveform using PCA/clustering techniques [7]. Column 4 makes a comparison between the stimulated action potential waveform (shown for a number of iterations-solid grey line) and the averaged waveform of the spontaneous neuron identified on the same electrode (dashed black line). The positions of neurons 1 and 2 are shown in figure 8(a), with the location of the associated spontaneous neurons ' $a$ ' and ' $b$ ' shown in figure 8(b).

microelectrodes, are commonly used to stimulate individual neurons or larger structures. Microelectrode array technologies and sophisticated electronics have been combined to achieve high spatial and temporal resolution, in vitro extracellular stimulation of neurons and the recording of their responses over a large area [29]. In acute slices the micro-needle array offers the advantage of close proximity to neurons, within a locally intact network, thereby increasing the efficiency for stimulation relative to that achievable with planar electrodes. The results in this section are taken from a single preparation of cortex from a p18 rat. The tissue was prepared as outlined in section 2.3 and once it became active, spontaneous activity was recorded for $30 \mathrm{~min}$. The following electrical stimulation scan was then run. A range of current amplitudes from 0.4 to $4 \mu \mathrm{A}$ with $10 \%$ increments was applied to each electrode (50 iterations for each current level). The current pulse was triphasic and charge balanced with duration of $50 \mu \mathrm{s} /$ phase, and with relative amplitudes for each phase of 2:-3:1 (see figure 7 , leftmost column). Triphasic pulses were used to reduce the electrical artefact [23]. The negative of the current amplitude of the second phase is used to specify the pulse current. 
(a)

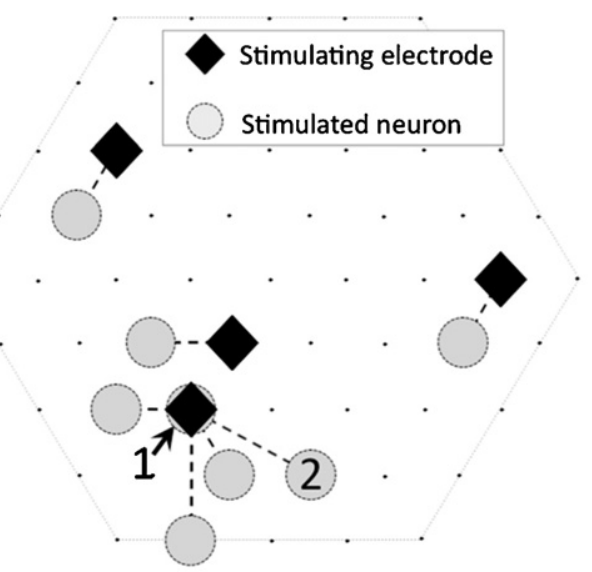

(b)

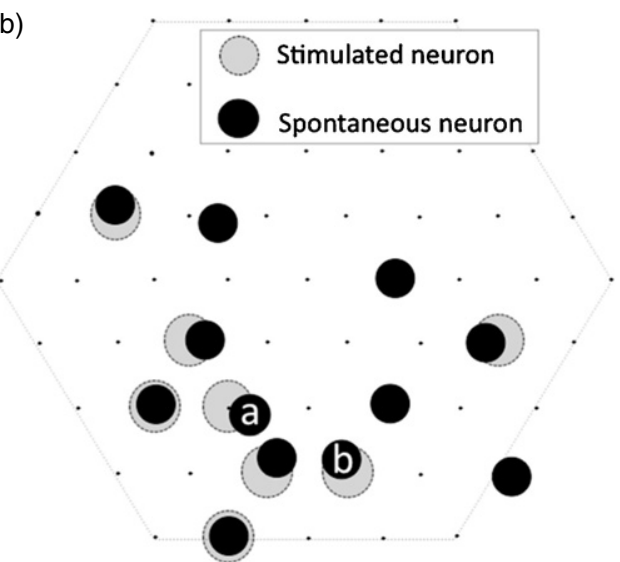

Figure 8. (a) The positions of eight electrically stimulated neurons. The positions of the stimulating electrodes are marked with a black diamond and are connected to the neurons they stimulate by a dashed line. (b) The positions of 12 spontaneously active neurons (black circles) found from a 30 min recording made prior to the stimulation experiment. These are overlaid with the positions of the stimulated neurons.
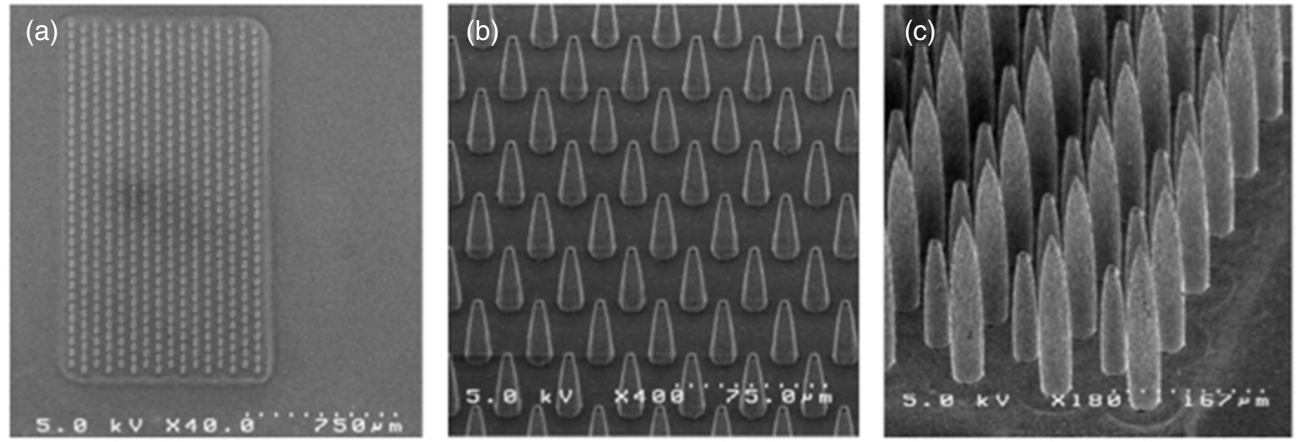

Figure 9. SEM images showing mechanical samples of future possibilities for the micro-needle array technology with (a) a large area $\left(1 \times 2 \mathrm{~mm}^{2}\right), 512$-needle array, (b) a higher density, $30 \mu \mathrm{m}$ spaced array and (c) a multi-length needle array.

To identify stimulated neurons, the recorded data from the stimulation experiment is organized in to $5 \mathrm{~ms}$ time periods taken from $50 \mu$ s following the end of each stimulation pulse. For each electrode and each stimulation current amplitude, the responses to the 50 iterations of that amplitude are overlaid in the same plot. These data are analysed to observe evidence of stimulation [30]. Figure 7 shows the triphasic stimulation pulses and the corresponding recorded signals, indicating stimulation of two identified neurons (neurons 1 and 2 corresponding to those labelled in figure 8(a)). For these examples, the stimulation efficiency was less than $100 \%$. Each plot in column 2 shows clearly two distinct clusters of waveforms: in red are the artefact waveforms (unsuccessful stimulation trials) and in blue are the artefacts plus elicited spike waveforms (successful stimulation trials). A comparison of successful stimulation trials with the averaged waveform of a spontaneous neuron found on the same electrode is also shown. These are seen to match well for both neurons. The spontaneous neurons are labelled ' $a$ ' and ' $b$ ' in figure 8(b) and correspond with stimulated neurons 1 and 2 respectively. It should be noted that this close comparison of waveforms is not always the case, which could perhaps be attributed to contamination during artefact subtraction or stimulation of a neuron that has no spontaneous activity.

Both the spontaneous and stimulation data were analysed to identify neurons. Figure 8(a) shows the positions (i.e. position of electrode on which the neuron's response is recorded) of the 8 neurons that were stimulated in this preparation. A line connects the electrode responsible for stimulating each neuron. From the spontaneous data, 12 neurons were identified and these are plotted in figure 8(b). They are overlaid on to the positions of the stimulated neurons.

\section{Discussion}

A challenging fabrication process was developed and successfully produced robust and re-usable arrays of 61 hexagonally close-packed micro-needles. The particular process developed is versatile and can be adapted to accommodate larger area, higher density arrays and multiple length needles on a single array (see figure 9).

Increasing the number of electrodes without compromising spatial resolution is of immediate interest. It is expected that an array of $\sim 500$ electrodes, hexagonally close-packed with $60 \mu \mathrm{m}$ inter-needle spacing (as shown in figure 9(a)), could record from an order of magnitude more neurons than the 61-needle array reported on here. The ability to simultaneously record from hundreds of neurons could be critical to further understanding connectivity in local networks. Whilst the process for micro-needle fabrication is versatile, it requires adjustments to produce devices with hundreds of needles. For 
example, the pitch of the bond pads requires the device to be directly wire-bonded to the readout electronics (the pitch is too high for use with $z$-axis connectors as with the 61-needle device). To enable this, through-silicon vias are required to connect the tungsten readout from each needle (on the backside of the device) to an aluminium bond pad on the front-side of the device and this requires some process development. Also, to have a uniform process over hundreds of needles, the deep reactive ion silicon etch and the thermal oxidation steps, in particular, have to be tightly controlled. In devices that increase spatial resolution and/or area coverage, higher resolution tungsten readout is required (e.g. a device with 512needles and $30 \mu \mathrm{m}$ hexagonally close-packed spacing has a minimum feature size of $1 \mu \mathrm{m}$ ) and is achievable with more advanced lithographic techniques. Arrays with multiple length needles are possible using the fabrication techniques outlined, though etch parameters (such as hole diameter and etch time) will have to be chosen carefully. The fundamentals of the fabrication process described in this paper are transferrable to future generations of micro-needle technology. Another future generation of this device could be a micro-needle array for in vivo applications. Ultimately, the ideal method for brain studies is in vivo, however, technology (in terms of the combination of spatial and temporal resolution) is limited for this preparation. Whilst, commercially available devices do exist $[19,31]$ and are very effective in the field, the high-density micro-needle array presented here, if appropriately modified, could be used, for example, to record from superficial cortical layers-potentially a rewarding endeavour with a dense electrode array. Substantial changes would be required to be made to the physical set-up of the electronics and the layout of the micro-needle array; however, they are realistic and are a potential future direction of this work.

A further point about the micro-needle recordings is worth noting. It can be seen in figure 8 that no neurons were found (from stimulated or spontaneous activity) on the top-right portion of the micro-needle array. Most likely this is from poor contact between the tissue and the array. The experimental procedure, in particular the tools used for weighting the tissue to the array, and also for tissue slicing, has since been improved. Therefore improvements in the number of neurons identified, coverage of the array, and signal to noise ratio (on both types of arrays) are expected as a consequence.

A noticeable feature of the EIs recorded from the microneedle array is their limited spread across, at most, seven or eight electrodes neighbouring the seed electrode (see figure 6). This EI geometry is different from the EIs recorded from the two-dimensional layout of ganglion cells in the retina [7] and could be attributed to a larger proportion of cells having myelinated axons (retinal ganglia are myelinated outside the region studied in [7]) or to the intrinsically three-dimensional nature of networks of neurons in the brain. To improve the spatial resolution and information content of the EIs from three-dimensional cortical structures, we plan to fabricate micro-needle arrays with a higher spatial density of electrodes, larger active area, and multiple-length needles as shown in figure 9.

As verification that higher density arrays are much more effective at detecting neurons, a comparison of the neuron identification analysis for 60 and $120 \mu \mathrm{m}$ spaced arrays was made in section 3.3 (figure 4). The most obvious result is that the number of neurons detected on the lower density array is significantly less. This is a consequence of the very localized detection of neurons in acute slices as well as the loss of information on a neuron from neighbouring electrodes. To demonstrate: from the preparation described in section 3 , 19 neurons and 6 neurons were found, respectively, from the 60 and $120 \mu \mathrm{m}$ analysis of the spontaneous activity of the same 30 min dataset. Of the 13 neurons not detected by the lower density array, 12 had the seed electrode identified on an electrode that was not used in the sub-sampled analysis, and insufficient spike amplitude to be above the threshold on any of the other sub-sampled electrodes. The remaining one neuron (see $\mathrm{A}$ in figure 4) was identified on an electrode used in the sub-sampled analysis but was discarded since there were not enough data to separate the neuron from background activity in the PCA analysis. An example of the difficulty in locating the position of a neuron, which lies outside of the array boundary, is shown by neuron B in figure 4 . The two analyses position the same neuron $\sim 100 \mu \mathrm{m}$ apart. Whilst the loss of information, as a result of the neuron not being surrounded by electrodes, makes the predicted position of the neuron unreliable for both analyses, the large separation is due to the fact that there is even less information from the $120 \mu \mathrm{m}$ analysis to constrain the neuron's location.

The final aspect of this study was to determine the microneedle technology's ability to electrically stimulate individual neurons in acute slices. The overall, positive result of the experiments performed was that this technology could, with low current amplitudes, elicit action potentials from several (up to 8) individual neurons in a single preparation. In these experiments it was found that for different neurons the stimulation threshold varied between 1.1 and $2.9 \mu \mathrm{A}$. The variation in thresholds could perhaps be due to the unknown electrode-neuron distance in the $z$-plane or differences in cell type. This result warrants further investigation perhaps using devices with the increased number of 512 needles (the second generation micro-needle device which is currently being developed) combined with imaging of the anatomy of the slice using fluorescent microscopy. This system is expected to be a very useful tool for future neural stimulation studies.

\section{Conclusions}

As an important in vitro model of the brain, it is important to study network behaviour in acute slices. The challenge for microelectrode arrays, however, is bypassing the dead layer of tissue that exists at the surface of an acute slice as a result of the preparation. In our attempts, planar arrays have not been entirely successful in achieving individual neuronal recordings with good signal to noise ratio from an undamaged layer of tissue. One approach is to make arrays of micro-needle electrodes that penetrate past this damaged layer and can record and stimulate extracellular neural activity in the healthy interior volume of the cut slice. In this paper, the fabrication and characterization of such a device were detailed. The novelty of the system described compared to 
current device of this nature lies in the high spatial and temporal resolution recording and electrical stimulation of single unit activity that it affords. The process developed has the versatility to incorporate up to hundreds of needles on a single array, higher densities $(\sim 30 \mu \mathrm{m}$ hexagonally closepacked) and multiple length needles ranging between 5 to $250 \mu \mathrm{m}$. As a first result, this state-of-the-art, first generation array of 61 hexagonally close-packed micro-needles was shown to successfully record action potentials from neurons ( $\sim 19$ neurons across $0.13 \mathrm{~mm}^{2}$ electrode array area) in acute cortical slices. Experiments ran with excellent signal to noise ratio (up to $\sim 15: 1$ ) and good coverage of the spiking activity over the array, with typical extracellular signal amplitudes of $30-110 \mu \mathrm{V}$. Individual neurons were stimulated with triphasic $50 \mu \mathrm{s} /$ phase charge-balanced current pulses, with thresholds from $\sim 1 \mu \mathrm{A}$. Proof of principle microfabrication processing shows the possibility of a second-generation microneedle array with multiple length needles to record from a volume of tissue and with 512 electrodes. The work reported in this paper represents a significant advancement in technologies for studying acute networks of neurons in vitro with an unprecedented combination of spatial and temporal resolutions.

\section{Acknowledgments}

Funding for this work was provided by a EPSRC grant EP/E039731/1 (DEG), the McKnight Foundation and NSF grants IIS-0904413 and PHY-0750525 (AML), NSF grant IIS0904912 (JMB), a Burroughs Welcome Fund Career Award at the Scientific Interface (AS), a Polish Ministry of Science and Higher Education grants for scientific research (WD) and RCUK funded SU2P Fellowship EP/G042446/1 (KM). The semiconductor device fabrication was done at the Stanford Nanofabrication Facility, Stanford University and the James Watt Nanofabrication Centre, University of Glasgow. The Queen's University, Belfast, was used for LPCVD tungsten and polysilicon depositions. We thank Sergei Kachiguine for technical contributions.

\section{References}

[1] Field G D 2010 Functional connectivity in the retina at the resolution of photoreceptors Nature $467673-7$

[2] Blankenship A G and Feller M B 2010 Mechanisms underlying spontaneous patterned activity in developing neural circuits Nature Rev. Neurosci. 11 18-29

[3] Malpass K 2012 Neurodegenerative disease: modelling connectivity networks of the brain-can neurodegeneration and dementia progression be predicted? Nature Rev. Neurol. 8237

[4] Zhou J, Gennatas E D, Kramer J H, Miller B L and Seeley W W 2012 Predicting regional neurodegeneration from the healthy brain functional connectome Neuron 73 1216-27

[5] Ebner T J and Chen G 1995 Use of voltage-sensitive dyes and optical recordings in the central nervous system Prog. Neurobiol. 46 463-506
[6] Grewe B F, Langer D, Kasper H, Kampa B M and Helmchen F 2010 High-speed in vivo calcium imaging reveals neuronal network activity with near-millisecond precision Nature Methods 7 399-405

[7] Litke A M et al 2004 What does the eye tell the brain?: Development of a system for the large-scale recording of retinal output activity IEEE Trans. Nucl. Sci. 51 1434-40

[8] Jimbo Y and Kawana A 1992 Electrical stimulation and recording from cultured neurons using a planar electrode array Bioelectrochem. Bioenerg. 29 193-204

[9] Gandolfo M, Maccione A, Tedesco M, Martinoia S and Berdondini L 2010 J. Neural Eng. 7056001

[10] Egert U, Schlosshauer B, Fennrich S, Nisch W, Fejtl M, Knott T, Muller T and Hammerle H 1998 A novel organotypic long-term culture of the rat hippocampus on substrate-integrated multielectrode arrays Brain Res. Protoc. 2 229-42

[11] Tang A et al 2008 A maximum entropy model applied to spatial and temporal correlations from cortical networks in vitro J. Neurosci. 28 505-18

[12] Egert U, Heck D and Aersten A 2002 Two dimensional monitoring of spiking networks in acute brain slices Exp. Brain Res. 142 268-74

[13] Wheeler B C and Novak J L 1986 Current source density estimation using microelectrode array data from the hippocampal slice preparation IEEE Trans. Biomed. Eng. 33 1204-12

[14] Oka H, Shimono K, Ogawa R, Sugihara H and Taketani M 1999 A new planar multielectrode array for extracellular recording: application to hippocampal acute slice J. Neurosci. Methods 93 61-7

[15] Castaneda-Castellanos D R, Flint A C and Kriegstein A R 2006 Blind patch clamp recordings in embryonic and adult mammalian brain slices Nature Protoc. 1 532-42

[16] Davie J T, Kole H P M, Letzkus J J, Rancz E A, Spruston N, Stuart G J and Hausser M 2006 Dendritic patch-clamp recording Nature Protoc. 1 1235-47

[17] Rajaraman S, McClain M A, Choi S, Ross J D, DeWeerth S P, LaPlaca M C and Allen M J 2011 Three-dimensional metal transfer micromolded microelectrode arrays for in vitro brain slice recordings $J$. Microelectromech. Syst. 20 396-409

[18] Kibler A B, Jamieson B G and Durand D M 2012 A high aspect ratio microelectrode array for mapping neural activity in vitro J. Neurosci. Methods 204 296-305

[19] Nordhausen C T, Rousche P J and Normann R A 1994 Optimizing recording capabilities of the Utah Intracortical Electrode Array Brain Res. 637 27-36

[20] Charvet $\mathrm{G}$ et al 2010 BioMEA $^{\mathrm{TM}}$ : a versatile high-density 3D microelectrode array system using integrated electronics Biosens. Bioelectron. 25 1889-96

[21] Heuschkel MO, Fejtl M, Raggenbass M, Bertrand D and Renaud P 2002 A three-dimensional multi-electrode array for multi-site stimulation and recording in acute brain slices J. Neurosci. Methods 114 135-48

[22] Hottowy P, Dabrowski W, Kachiguine S, Skoczen A, Fiutowski T, Sher A, Rydgier P, Grillo A A and Litke A M 2008 An MEA-based system for multichannel, low artifact stimulation and recording of neural activity Proc. 6th Int. Meeting on Substrate-Integrated Microelectrode Arrays pp 259-62

[23] Hottowy P, Skoczen A, Gunning D E, Kachiguine S, Mathieson K, Sher A, Wiacek P, Litke A M and Dabrowski W 2012 Properties and application of a multichannel integrated circuit for low-artifact, patterned electrical stimulation of neural tissue J. Neural Eng. 9066005

[24] Grybos P, Dabrowski W, Hottowy P, Fiutowski T and Bielewicz B 2006 NEUROPLAT64-low noise CMOS 
integrated circuit for neural recording applications Proc. 5th Int. Meeting on Substrate-Integrated Microelectrode Arrays pp 208-9

[25] Hottowy P, Dabrowski W, Skoczen A and Wiacek P 2008 An integrated multichannel waveform generator for large-scale spatio-temporal stimulation of neural tissue Analog Integr. Circuits Signal Process. 55 239-48

[26] Wu J-y, Guan L and Tsau Y 1999 Propagating activation during oscillations and evoked responses in neocortical slices J. Neurosci. 19 5005-15 PMID: 10366633

[27] Chen W, Hobbs J P, Tang A and Beggs J M 2010 A few strong connections:optimizing information retention in neuronal avalanches BMC Neurosci. 11 1-14
[28] Agmon A and Connors B W 1991 Thalamocortical responses of mouse somatosensory (barrel) cortex in vitro Neuroscience 41 365-79

[29] Merrill D R, Bikson M and Jefferys J G R 2005 Electrical stimulation of excitable tissue: design of efficacious and safe protocols J. Neurosci. Methods 141 171-98

[30] Sekirnjak C, Hottowy P, Sher A, Dabrowski W, Litke A M and Chichilnisky E J 2006 Electrical stimulation of mammalian retinal ganglion cells with multielectrode arrays J. Neurophysiol. 95 3311-27

[31] Mizuseki K, Diba K, Pastalkova E. and Buzsaki G 2011 Hippocampal CA1 pyramidal cells form functionally distinct sublayers Nature Neurosci. 14 1174-81 